\title{
A generalized Borel-reducibility counterpart of Shelah's Main Gap Theorem
}

\section{Hyttinen, Tapani}

2017-05

Hyttinen, T, Kulikov, V \& Moreno, M 2017 , ' A generalized Borel-reducibility counterpart of Shelah's Main Gap Theorem ' , Archive for Mathematical Logic , vol. 56 , no. 3-4 , pp. 175-185 . https://doi.org/10.1007/s00153-017-0521-3

http://hdl.handle.net/10138/307405

https://doi.org/10.1007/s00153-017-0521-3

cc_by_nc_sa

acceptedVersion

Downloaded from Helda, University of Helsinki institutional repository.

This is an electronic reprint of the original article.

This reprint may differ from the original in pagination and typographic detail.

Please cite the original version. 


\title{
A Borel-reducibility Counterpart of Shelah's Main Gap Theorem
}

\author{
Tapani Hyttinen, Vadim Kulikov, Miguel Moreno \\ University of Helsinki
}

\begin{abstract}
We study the Borel-reducibility of isomorphism relations of complete first order theories and show the consistency of the following: For all such theories $\mathrm{T}$ and $\mathrm{T}^{\prime}$, if $\mathrm{T}$ is classifiable and $\mathrm{T}^{\prime}$ is not, then the isomorphism of models of $\mathrm{T}^{\prime}$ is strictly above the isomorphism of models of $\mathrm{T}$ with respect to Borel-reducibility. In fact, we can also ensure that a range of equivalence relations modulo various non-stationary ideals are strictly between those isomorphism relations. The isomorphism relations are considered on models of some fixed uncountable cardinality obeying certain restrictions.
\end{abstract}

\section{Introduction}

Throughout this article we assume that $\kappa$ is an uncountable cardinal that satisfies $\kappa^{<\kappa}=\kappa$. The generalized Baire space is the set $\kappa^{\kappa}$ with the bounded topology. For every $\zeta \in \kappa^{<\kappa}$, the set

$$
[\zeta]=\left\{\eta \in \kappa^{\kappa} \mid \zeta \subset \eta\right\}
$$

is a basic open set. The open sets are of the form $U X$ where $X$ is a collection of basic open sets. The collection of $\kappa$-Borel subsets of $\kappa^{\kappa}$ is the smallest set which contains the basic open sets and is closed under unions and intersections, both of length $\kappa$. A $\kappa$-Borel set is any element of this collection. We usually omit the prefix " $\kappa-$ ". In [Vau74] Vought studied this topology in the case $\kappa=\omega_{1}$ assuming CH and proved the following:

Theorem. A set $B \subset \omega_{1}^{\omega_{1}}$ is Borel and closed under permutations if and only if there is a sentence $\varphi$ in $L_{\omega_{1}^{+} \omega_{1}}$ such that $B=\left\{\eta \mid \mathcal{A}_{\eta} \models \varphi\right\}$.

This result was generalized in [FHK14] to arbitrary $\kappa$ that satisfies $\kappa^{<\kappa}=\kappa$. Mekler and Väänänen continued the study of this topology in [MV93].

We will work with the subspace $2^{\kappa}$ with the relative subspace topology. A function $f: 2^{\kappa} \rightarrow 2^{\kappa}$ is Borel, if for every open set $A \subseteq 2^{\kappa}$ the inverse image $f^{-1}[A]$ is a Borel subset of $2^{\kappa}$. Let $E_{1}$ and $E_{2}$ be equivalence relations on $2^{\kappa}$. We say that $E_{1}$ is Borel reducible to $E_{2}$, if there is a Borel function $f: 2^{\kappa} \rightarrow 2^{\kappa}$ that satisfies $(x, y) \in E_{1} \Leftrightarrow(f(x), f(y)) \in E_{2}$. We call $f$ a reduction of $E_{1}$ to $E_{2}$. This is denoted by $E_{1} \leq_{B} E_{2}$ and if $f$ is continuous, then we say that $E_{1}$ is continuously reducible to $E_{2}$ and this is denoted by $E_{1} \leq_{c} E_{2}$.

The following is a standard way to code structures with domain $\kappa$ with elements of $2^{\kappa}$. To define it, fix a countable relational vocabulary $\mathcal{L}=\left\{P_{n} \mid n<\omega\right\}$. 
Definition 1.1. Fix a bijection $\pi: \kappa^{<\omega} \rightarrow \kappa$. For every $\eta \in 2^{\kappa}$ define the $\mathcal{L}$-structure $\mathcal{A}_{\eta}$ with domain $\kappa$ as follows: For every relation $P_{m}$ with arity $n$, every tuple $\left(a_{1}, a_{2}, \ldots, a_{n}\right)$ in $\kappa^{n}$ satisfies

$$
\left(a_{1}, a_{2}, \ldots, a_{n}\right) \in P_{m}^{\mathcal{A}_{\eta}} \Longleftrightarrow \eta\left(\pi\left(m, a_{1}, a_{2}, \ldots, a_{n}\right)\right)=1 .
$$

Note that for every $\mathcal{L}$-structure $\mathcal{A}$ there exists $\eta \in 2^{\kappa}$ with $\mathcal{A}=\mathcal{A}_{\eta}$. For club many $\alpha<\kappa$ we can also code the $\mathcal{L}$-structures with domain $\alpha$ :

Definition 1.2. Denote by $C_{\pi}$ the club $\left\{\alpha<\kappa \mid \pi\left[\alpha^{<\omega}\right] \subseteq \alpha\right\}$. For every $\eta \in 2^{\kappa}$ and every $\alpha \in C_{\pi}$ define the structure $\mathcal{A}_{\eta \mid \alpha}$ with domain $\alpha$ as follows: For every relation $P_{m}$ with arity $n$, every tuple $\left(a_{1}, a_{2}, \ldots, a_{n}\right)$ in $\alpha^{n}$ satisfies

$$
\left(a_{1}, a_{2}, \ldots, a_{n}\right) \in P_{m}^{\mathcal{A}_{\eta \uparrow \alpha}} \Longleftrightarrow \eta \uparrow_{\alpha}\left(\pi\left(m, a_{1}, a_{2}, \ldots, a_{n}\right)\right)=1 .
$$

For every $\alpha \in C_{\pi}$ and every $X \subseteq \alpha$ we will denote the structure $\mathcal{A}_{F}$ by $\mathcal{A}_{X}$, where $F$ is the characteristic function of $X$. We will work with two equivalence relations on $2^{\kappa}$ : the isomorphism relation and the equivalence modulo the non-stationary ideal.

Definition 1.3 (The isomorphism relation). Assume $T$ is a complete first order theory in a countable vocabulary. We define $\cong{ }_{T}^{\mathcal{K}}$ as the relation

$$
\left\{(\eta, \xi) \in 2^{\kappa} \times 2^{\kappa} \mid\left(\mathcal{A}_{\eta} \models T, \mathcal{A}_{\xi} \models T, \mathcal{A}_{\eta} \cong \mathcal{A}_{\xi}\right) \text { or }\left(\mathcal{A}_{\eta} \not \models T, \mathcal{A}_{\xi} \not \models T\right)\right\}
$$

We will omit the superscript " $\kappa^{\prime}$ in $\cong_{T}^{\kappa}$ when it is clear from the context. For every first order theory $T$ in a countable vocabulary there is an isomorphism relation associated with $T, \cong_{T}^{\kappa}$. For every stationary $X \subset \kappa$, we define an equivalence relation modulo the non-stationary ideal associated with $X$ :

Definition 1.4. For every $X \subset \kappa$ stationary, we define $E_{X}$ as the relation

$$
E_{X}=\left\{(\eta, \xi) \in 2^{\kappa} \times 2^{\kappa} \mid\left(\eta^{-1}[1] \triangle \xi^{-1}[1]\right) \cap X \text { is not stationary }\right\}
$$

where $\triangle$ denotes the symmetric difference.

For every regular cardinal $\mu<\kappa$ denote $\{\alpha<\kappa \mid c f(\alpha)=\mu\}$ by $S_{\mu}^{\kappa}$. A set $C$ is $\mu$-club if it is ubounded and closed under $\mu$-limits, i.e. if $S_{\mu}^{\kappa} \backslash C$ is non-stationary. Accordingly, we will denote the equivalence relation $E_{X}$ for $X=S_{\mu}^{\kappa}$ by $E_{\mu \text {-club }}^{2}$. Note that $(f, g) \in E_{\mu \text {-club }}^{2}$ if and only if the set $\{\alpha<\kappa \mid f(\alpha)=g(\alpha)\}$ contains a $\mu$-club.

\section{Reduction to $E_{X}$}

Classifiable theories (superstable with NOTOP and NDOP) have a close connection to the EhrenfeuchtFraïssé games (EF-games for short). We will use them to study the reducibility of the isomorphism relation of classifiable theories. The following definition is from [HM15, Def 2.3]:

Definition 2.1 (The Ehrenfeucht-Fraïssé game). Fix an enumeration $\left\{X_{\gamma}\right\}_{\gamma<\kappa}$ of the elements of $\mathcal{P}_{\mathcal{K}}(\mathcal{K})$ and an enumeration $\left\{f_{\gamma}\right\}_{\gamma<\kappa}$ of all the functions with both the domain and range in $\mathcal{P}_{\kappa}(\kappa)$. For every $\alpha \leqslant \kappa$ the game $\mathrm{EF}_{\omega}^{\alpha}\left(\mathcal{A} \uparrow_{\alpha}, \mathcal{B} \uparrow_{\alpha}\right)$ on the restrictions $\mathcal{A}\lceil\alpha$ and $\mathcal{B}\lceil\alpha$ of the structures $\mathcal{A}$ and $\mathcal{B}$ with domain $\kappa$ is defined as follows: In the $n$-th move, first I chooses an ordinal $\beta_{n}<\alpha$ such that $X_{\beta_{n}} \subset \alpha$ and $X_{\beta_{n-1}} \subseteq X_{\beta_{n}}$. Then II chooses an ordinal $\theta_{n}<\alpha$ such that $\operatorname{dom}\left(f_{\theta_{n}}\right), \operatorname{ran}\left(f_{\theta_{n}}\right) \subset \alpha, X_{\beta_{n}} \subseteq \operatorname{dom}\left(f_{\theta_{n}}\right) \cap \operatorname{ran}\left(f_{\theta_{n}}\right)$ and $f_{\theta_{n-1}} \subseteq f_{\theta_{n}}$ (if $n=0$ then $X_{\beta_{n-1}}=\varnothing$ and $\left.f_{\theta_{n-1}}=\varnothing\right)$. The game ends after $\omega$ moves. Player II wins if $\bigcup_{i<\omega} f_{\theta_{i}}: A \uparrow_{\alpha} \rightarrow B \uparrow_{\alpha}$ is a partial isomorphism. Otherwise player I wins. If $\alpha=\kappa$ then this is the same as the standard EF-game which is usually denoted by $\mathrm{EF}_{\omega}^{\kappa}$.

When a player $P$ has a winning strategy in a game $G$, we denote it by $P \uparrow G$. 
The following lemma is proved in [HM15, Lemma 2.4] and is used in the main result of this section which in turn is central to the main theorem of this paper.

Lemma 2.2. If $\mathcal{A}$ and $\mathcal{B}$ are structures with domain $\kappa$, then

- II $\uparrow \mathrm{EF}_{\omega}^{\kappa}(\mathcal{A}, \mathcal{B}) \Longleftrightarrow \mathrm{II} \uparrow \mathrm{EF}_{\omega}^{\alpha}\left(\mathcal{A} \uparrow_{\alpha}, \mathcal{B} \uparrow_{\alpha}\right)$ for club-many $\alpha$,

- $\mathbf{I} \uparrow \mathrm{EF}_{\omega}^{\kappa}(\mathcal{A}, \mathcal{B}) \Longleftrightarrow \mathbf{I} \uparrow \mathrm{EF}_{\omega}^{\alpha}\left(\mathcal{A} \uparrow_{\alpha}, \mathcal{B} \uparrow_{\alpha}\right)$ for club-many $\alpha$.

Remark 1. In [HM15, Lemma 2.7] it was proved that there exists a club $C_{\mathrm{EF}}$ of $\alpha$ such that the relation defined by the game

$$
\left\{(\mathcal{A}, \mathcal{B}) \mid \mathbf{I I} \uparrow \operatorname{EF}_{\omega}^{\alpha}\left(\mathcal{A}\left\lceil_{\alpha}, \mathcal{B} \Upsilon_{\alpha}\right)\right\}\right.
$$

is an equivalence relation.

Remark 2. Shelah proved in [She90], that if $T$ is classifiable then every two models of $T$ that are $L_{\infty, \kappa^{-}}$ equivalent are isomorphic. On the other hand $L_{\infty, \kappa}$-equivalence is equivalent to $\mathrm{EF}_{\omega}^{\kappa}$-equivalence. So for every two models $\mathcal{A}$ and $\mathcal{B}$ of $T$ we have $\operatorname{II} \uparrow \mathrm{EF}_{\omega}^{\kappa}(\mathcal{A}, \mathcal{B}) \Longleftrightarrow \mathcal{A} \cong \mathcal{B}$ and $\mathbf{I} \uparrow \operatorname{EF}_{\omega}^{\kappa}(\mathcal{A}, \mathcal{B}) \Longleftrightarrow \mathcal{A} ¥ \mathcal{B}$.

Lemma 2.3. Assume $T$ is a classifiable theory and $\mu<\kappa$ is a regular cardinal. If $\nabla_{\mathcal{K}}(X)$ holds then $\cong_{T}^{\kappa}$ is continuously reducible to $E_{X}$.

Proof. Let $\left\{S_{\alpha} \mid \alpha \in X\right\}$ be a sequence testifying $\diamond_{\kappa}(X)$ and define the function $\mathcal{F}: 2^{\kappa} \rightarrow 2^{\kappa}$ by

$$
\mathcal{F}(\eta)(\alpha)= \begin{cases}1 & \text { if } \alpha \in X \cap C_{\pi} \cap C_{E F}, \mathbf{I I} \uparrow E F_{\omega}^{\kappa}\left(\mathcal { A } _ { \eta } \lceil _ { \alpha } , \mathcal { A } _ { S _ { \alpha } } ) \text { and } \mathcal { A } _ { \eta } \left\lceil_{\alpha} \models T\right.\right. \\ 0 & \text { otherwise. }\end{cases}
$$

Let us show that $\mathcal{F}$ is a reduction of $\cong_{T}$ to $E_{X}$, i.e. for every $\eta, \xi \in 2^{\kappa},(\eta, \xi) \in \cong_{T}$ if and only if $(\mathcal{F}(\eta), \mathcal{F}(\xi)) \in E_{X}$. Notice that when $\alpha \in C_{\pi}$, the structure $\mathcal{A}_{\eta\lceil\alpha}$ is defined and equals $\mathcal{A}_{\eta}\left\lceil_{\alpha}\right.$.

Consider first the direction from left to right. Suppose first that $\mathcal{A}_{\eta}$ and $\mathcal{A}_{\xi}$ are models of $T$ and $\mathcal{A}_{\eta} \cong \mathcal{A}_{\xi}$. Since $\mathcal{A}_{\eta} \cong \mathcal{A}_{\xi}$, we have II $\uparrow \mathrm{EF}_{\omega}^{\kappa}\left(\mathcal{A}_{\eta}, \mathcal{A}_{\xi}\right)$. By Lemma 2.2 there is a club $C$ such that II $\uparrow \mathrm{EF}_{\omega}^{\alpha}\left(\mathcal{A}_{\eta} \Upsilon_{\alpha}, \mathcal{A}_{\xi} \Upsilon_{\alpha}\right)$ for every $\alpha$ in $C$. Since the set $\left\{\alpha<\kappa \mid \mathcal{A}_{\eta} \Upsilon_{\alpha} \models T, \mathcal{A}_{\xi} \Upsilon_{\alpha} \models T\right\}$ contains a club, we can assume that every $\alpha \in C$ satisfies $\mathcal{A}_{\eta} \uparrow_{\alpha} \models T$ and $\mathcal{A}_{\xi} \uparrow_{\alpha} \models T$. If $\alpha \in C$ is such that $\mathcal{F}(\eta)(\alpha)=1$, then II $\uparrow \mathrm{EF}_{\omega}^{\alpha}\left(\mathcal{A}_{\eta} \uparrow_{\alpha}, \mathcal{A}_{S_{\alpha}}\right)$. Since II $\uparrow \mathrm{EF}_{\omega}^{\alpha}\left(\mathcal{A}_{\eta} \uparrow_{\alpha}, \mathcal{A}_{\xi} \uparrow_{\alpha}\right)$ and $\alpha \in C_{E F}$, we can conclude that II $\uparrow \mathrm{EF}_{\omega}^{\alpha}\left(\mathcal{A}_{\xi}\left\lceil\alpha, \mathcal{A}_{S_{\alpha}}\right)\right.$. Therefore for every $\alpha \in C, \mathcal{F}(\eta)(\alpha)=1$ implies $\mathcal{F}(\xi)(\alpha)=1$. Using the same argument it can be shown that for every $\alpha \in C, \mathcal{F}(\xi)(\alpha)=1$ implies $\mathcal{F}(\eta)(\alpha)=1$. Therefore $\mathcal{F}(\eta)$ and $\mathcal{F}(\xi)$ coincide in a club and $(\mathcal{F}(\eta), \mathcal{F}(\xi)) \in E_{X}$.

Let us now look at the case where $(\eta, \xi) \in \cong_{T}$ and $\mathcal{A}_{\eta}$ is not a model of $T$ (the case $T \not \models \mathcal{A}_{\xi}$ follows by symmetry). By the definition of $\cong_{T}$ we know that $\mathcal{A}_{\xi}$ is not a model of $T$ either, so there is $\varphi \in T$ such that $\mathcal{A}_{\eta} \models \neg \varphi$ and $\mathcal{A}_{\xi} \models \neg \varphi$. Further, there is a club $C$ such that for every $\alpha \in C$ we have $\left.\mathcal{A}_{\eta}\right|_{\alpha} \models \neg \varphi$ and $\mathcal{A}_{\xi} \uparrow_{\alpha}=\neg \varphi$. We conclude that for every $\alpha \in C$ we have that $\mathcal{A}_{\eta} \uparrow_{\alpha}$ and $\mathcal{A}_{\xi} \uparrow_{\alpha}$ are not models of $T$, and $\mathcal{F}(\eta)(\alpha)=\mathcal{F}(\xi)(\alpha)=0$, so $(\mathcal{F}(\eta), \mathcal{F}(\xi)) \in E_{X}$.

Let us now look at the direction from right to left. Suppose first that $\mathcal{A}_{\eta}$ and $\mathcal{A}_{\xi}$ are models of $T$, and $\mathcal{A}_{\eta} \neq \mathcal{A}_{\xi}$.

By Remark 2, we know that $\mathbf{I} \uparrow \mathrm{EF}_{\omega}^{\kappa}\left(\mathcal{A}_{\eta}, \mathcal{A}_{\xi}\right)$. By Lemma 2.2 there is a club $C$ of $\alpha$ with

$$
\mathbf{I} \uparrow \mathrm{EF}_{\omega}^{\alpha}\left(\mathcal { A } _ { \eta } \left\lceil_{\alpha}, \mathcal{A}_{\xi}\left\lceil_{\alpha}\right),\right.\right.
$$

$\mathcal{A}_{\xi\lceil\alpha} \models T$ and $\mathcal{A}_{\eta} \Upsilon_{\alpha} \models T$. 
Since $\left\{\alpha \in X \mid \eta \cap \alpha=S_{\alpha}\right\}$ is stationary by the definition of $\nabla_{\mathcal{K}}(X)$, also the set

$$
\left\{\alpha \in X \mid \eta \cap \alpha=S_{\alpha}\right\} \cap C_{\pi} \cap C_{E F}
$$

is stationary and every $\alpha$ in this set satisfies II $\uparrow \operatorname{EF}_{\omega}^{\kappa}\left(\mathcal{A}_{\eta} \uparrow_{\alpha}, \mathcal{A}_{S_{\alpha}}\right)$. Therefore

$$
C \cap\left\{\alpha \in X \mid \eta \cap \alpha=S_{\alpha}\right\} \cap C_{\pi} \cap C_{E F}
$$

is stationary and a subset of $\mathcal{F}(\eta)^{-1}\{1\} \triangle \mathcal{F}(\xi)^{-1}\{1\}$, where $\triangle$ denotes the symmetric difference. We conclude that $(\mathcal{F}(\eta), \mathcal{F}(\xi)) \notin E_{X}$.

Let us finally assume that $(\eta, \xi) \notin \cong_{T}$ and $\mathcal{A}_{\eta} \not \models T$ (the case $\mathcal{A}_{\xi} \not \models T$ follows by symmetry). Assume towards a contradiction that $(\mathcal{F}(\eta), \mathcal{F}(\xi)) \in E_{\mu \text {-club }}^{2}$. Let $C$ be a club that testifies $(\mathcal{F}(\eta), \mathcal{F}(\xi)) \in E_{\mu \text {-club }}^{2}$ i.e. $C \cap\left(\mathcal{F}(\eta)^{-1}[1] \triangle \mathcal{F}(\xi)^{-1}[1]\right) \cap X=\varnothing$. Since $\mathcal{A}_{\eta} \not \models T$, the set $\left\{\alpha<\mathcal{\kappa} \mid \mathcal{A}_{\eta}\lceil\alpha \mid \neq T\}\right.$ contains a club. Hence, we can assume that for every $\alpha \in C, \mathcal{A}_{\eta} \uparrow_{\alpha} \not \models T$ which implies that $\mathcal{F}(\eta)(\alpha)=0$ and $\mathcal{F}(\xi)(\alpha)=0$ for every $\alpha \in C$.

By the definition of $\cong_{T}, \mathcal{A}_{\eta} \not \models T$ implies $\mathcal{A}_{\xi} \models T$. Therefore the set $\left\{\alpha<\kappa \mid \mathcal{A}_{\xi}\lceil\alpha \models T\}\right.$ contains a club. So there is a club $C^{\prime}$ such that every $\alpha \in C^{\prime}$ satisfies $\mathcal{A}_{\xi} \Gamma_{\alpha} \models T$ and $\mathcal{F}(\xi)(\alpha)=0$. Since $\left\{\alpha \in X \mid \xi \cap \alpha=S_{\alpha}\right\}$ is stationary, again by the definition of $\diamond_{\mathcal{K}}(X)$, also $\left\{\alpha \in X \mid \eta \cap \alpha=S_{\alpha}\right\} \cap C_{\pi} \cap C_{E F}$ is stationary and every $\alpha$ in this set satisfies II $\uparrow \mathrm{EF}_{\omega}^{\kappa}\left(\mathcal{A}_{\eta} \uparrow_{\alpha}, \mathcal{A}_{S_{\alpha}}\right)$. Therefore,

$$
C^{\prime} \cap\left\{\alpha \in X \mid \xi \cap \alpha=S_{\alpha}\right\} \cap C_{\pi} \cap C_{E F} \neq \varnothing,
$$

a contradiction.

To show that $\mathcal{F}$ is continuous, let $\left[\eta \uparrow_{\alpha}\right]$ be a basic open set, $\xi \in \mathcal{F}^{-1}\left[\left[\eta \uparrow_{\alpha}\right]\right]$. Then $\xi \in\left[\xi \uparrow_{\alpha}\right]$ and $\left[\xi \Gamma_{\alpha}\right] \subseteq \mathcal{F}^{-1}\left[\left[\eta \uparrow_{\alpha}\right]\right]$. We conclude that $\mathcal{F}$ is continuous.

To define the reduction $\mathcal{F}$ it is not enough to use the isomorphism classes of the models $\mathcal{A}_{S_{\alpha}}$, as opposed to the equivalence classes of the relation defined by the EF-game. It is possible to construct two non-isomorphic models with domain $\kappa$ such that their restrictions to any $\alpha<\kappa$ are isomorphic. For example the models $\mathcal{M}=(\kappa, P)$ and $\mathcal{N}=(\kappa, Q)$, with $\kappa=\lambda^{+}$,

$$
P=\{\alpha<\kappa \mid \alpha=\beta+2 n, n \in \mathbb{N} \text { and } \beta \text { a limit ordinal }\}
$$

and

$$
Q=\{\alpha<\lambda \mid \alpha=\beta+2 n, n \in \mathbb{N} \text { and } \beta \text { a limit ordinal }\}
$$

are non-isomorphic but $\mathcal{M} \uparrow_{\alpha} \cong \mathcal{N} \uparrow_{\alpha}$ holds for every $\alpha<\kappa$.

The Borel reducibility of the isomorphism relation of classifiable theories was studied in [FHK14] and one of the main results is the following.

Theorem 2.4. ([FHK14, Thm 77]) If a first order theory $T$ is classifiable, then for all regular cardinals $\mu<\kappa$, $E_{\mu-c l u b}^{2} \mathbb{S}_{B} \cong{ }_{T}^{K}$.

Corollary 2.5. Assume that $\nabla_{\mathcal{K}}\left(S_{\mu}^{\kappa}\right)$ holds for all regular $\mu<\kappa$. If a first order theory $T$ is classifiable, then for all regular cardinals $\mu<\kappa$ we have $\cong_{T}^{\kappa} \leqslant_{c} E_{\mu-c l u b}^{2}$ and $E_{\mu-c l u b}^{2} \mathbb{E}_{B} \cong_{T}^{\kappa}$. 


\section{Non-classifiable Theories}

In [FHK14] the reducibility to the isomorphism of non-classifiable theories was studied. In particular the following two theorems were proved there:

Theorem 3.1. ([FHK14, Thm 79]) Suppose that $\kappa=\lambda^{+}=2^{\lambda}$ and $\lambda^{<\lambda}=\lambda$.

1. If $T$ is unstable or superstable with OTOP, then $E_{\lambda-c l u b}^{2} \leqslant_{c} \cong_{T}^{\kappa}$.

2. If $\lambda \geqslant 2^{\omega}$ and $T$ is superstable with DOP, then $E_{\lambda \text {-club }}^{2} \leqslant_{c} \cong_{T}^{\kappa}$.

Theorem 3.2. ([FHK14, Thm 86]) Suppose that for all $\gamma<\kappa, \gamma^{\omega}<\kappa$ and $T$ is a stable unsuperstable theory. Then $E_{\omega-c l u b}^{2} \leqslant_{c} \cong{ }_{T}^{k}$.

Clearly from Theorems 3.1 and 3.2 and Corollary 2.3 we obtain the following:

Theorem 3.3. Suppose that $\kappa=\lambda^{+}=2^{\lambda}, \lambda^{<\lambda}=\lambda$ and $\nabla_{\kappa}\left(S_{\lambda}^{\kappa}\right)$ holds.

1. If $T_{1}$ is classifiable and $T_{2}$ is unstable or superstable with OTOP, then $\cong_{T_{1}}^{\kappa} \leqslant_{c} \cong_{T_{2}}^{\kappa}$ and $\cong_{T_{2}}^{\kappa} \mathbb{B}_{B} \cong_{T_{1}}^{\kappa}$.

2. If $\lambda \geqslant 2^{\omega}, T_{1}$ is classifiable and $T_{2}$ is superstable with DOP, then $\cong_{T_{1}}^{\kappa} \leqslant_{c} \cong_{T_{2}}^{\kappa}$ and $\cong_{T_{2}}^{\kappa} \mathbb{k}_{B} \cong_{T_{1}}^{\kappa}$.

Theorem 3.4. Suppose that for all $\gamma<\kappa, \gamma^{\omega}<\kappa$ and $\nabla_{\kappa}\left(S_{\omega}^{\kappa}\right)$ holds. If $T_{1}$ is classifiable and $T_{2}$ is stable unsuperstable, then $\cong_{T_{1}}^{\kappa} \leqslant c \cong{ }_{T_{2}}^{\mathcal{K}}$ and $\cong_{T_{2}}^{\mathcal{K}} \mathbb{B}_{B} \cong{ }_{T_{1}}^{\mathcal{K}}$.

Corollary 3.5. Suppose $\kappa=\kappa^{<\kappa}=\lambda^{+}$and $\lambda^{\omega}=\lambda$. If $T_{1}$ is classifiable and $T_{2}$ is stable unsuperstable, then $\cong_{T_{1}}^{\kappa} \leqslant_{c} \cong_{T_{2}}^{\kappa}$ and $\cong_{T_{2}}^{\kappa} \mathbb{B}_{B} \cong_{T_{1}}^{\kappa}$.

Proof. In [She10] Shelah proved that if $\kappa=\lambda^{+}=2^{\lambda}$ and $S$ is a stationary subset of $\{\alpha<\kappa \mid c f(\alpha) \neq$ $c f(\lambda)\}$, then $\nabla_{\mathcal{K}}(S)$ holds. Since $\lambda^{\omega}=\lambda$, we have $c f(\lambda) \neq \omega$ and $\nabla_{\mathcal{K}}\left(S_{\omega}^{\kappa}\right)$ holds. On the other hand $\kappa=\lambda^{+}$and $\lambda^{\omega}=\lambda$ implies $\gamma^{\omega}<\kappa$ for all $\gamma<\kappa$. By Theorem 3.4 we conclude that if $T_{1}$ is a classifiable theory and $T_{2}$ is a stable unsuperstable theory, then $\cong_{T_{1}} \leqslant_{c} \cong_{T_{2}}$ and $\cong_{T_{2}} \mathbb{k}_{B} \cong_{T_{1}}$.

Theorem 3.6. Let $H(\kappa)$ be the following property: If $T$ is classifiable and $T^{\prime}$ not, then $\cong_{T}^{\kappa} \leqslant_{c} \cong_{T^{\prime}}^{k}$ and $\cong T_{T^{\prime}}^{k} \mathbb{B}_{B}$ $\cong_{T}^{\kappa}$. Suppose that $\kappa=\kappa^{<\kappa}=\lambda^{+}, 2^{\lambda}>2^{\omega}$ and $\lambda^{<\lambda}=\lambda$.

1. If $V=L$, then $H(\kappa)$ holds.

2. There is a $\kappa$-closed forcing notion $\mathbb{P}$ with the $\kappa^{+}$-c.c. which forces $H(\kappa)$.

Proof. 1. This follows from Theorems 3.3 and 3.4.

2. Let $\mathbb{P}$ be $\{f: X \rightarrow 2|X \subseteq \kappa| X \mid,<\kappa\}$ with the order $p \leqslant q$ if $q \subset p$. It is known that $\mathbb{P}$ has the $\kappa^{+}$-cc [Kun11, Lemma IV.7.5] and is $\kappa$-closed [Kun11, Lemma IV.7.14]. It is also known that $\mathbb{P}$ preserves cofinalities, cardinalities and subsets of $\kappa$ of size less than $\kappa$ [Kun11, Thm IV.7.9, Lemma IV.7.15]. Therefore, in $V[G], \kappa$ satisfies $\kappa=\kappa^{<\kappa}=\lambda^{+}=2^{\lambda}>2^{\omega}$ and $\lambda^{<\lambda}=\lambda$. It is known that $\mathbb{P}$ satisfies $\mathbb{1} \Vdash_{\mathbb{P}} \diamond_{k}\left(S_{\mu}^{\kappa}\right)$ for every regular cardinal $\mu<\kappa$. Therefore, by Theorems 3.3 and $3.4 H(\kappa)$ holds in $V[G]$.

Definition 3.7. 1. A tree $T$ is $a \kappa^{+}, \kappa$-tree if does not contain chains of length $\kappa$ and its cardinality is less than $\kappa^{+}$. It is closed if every chain has a unique supremum. 
2. A pair $(T, h)$ is a Borel ${ }^{*}$-code if $T$ is a closed $\kappa^{+}, \kappa$-tree and $h$ is a function with domain $T$ such that if $x \in T$ is a leaf, then $h(x)$ is a basic open set and otherwise $h(x) \in\{\cup, \cap\}$.

3. For an element $\eta \in 2^{\kappa}$ and a Borel ${ }^{*}$-code $(T, h)$, the Borel ${ }^{*}$-game $B^{*}(T, h, \eta)$ is played as follows. There are two players, $\mathbf{I}$ and $\mathbf{I I}$. The game starts from the root of $T$. At each move, if the game is at node $x \in T$ and $h(x)=\cap$, then $\mathbf{I}$ chooses an immediate successor $y$ of $x$ and the game continues from this $y$. If $h(x)=\cup$, then II makes the choice. At limits the game continues from the (unique) supremum of the previous moves by Player I. Finally, if $h(x)$ is a basic open set, then the game ends, and II wins if and only if $\eta \in h(x)$.

4. A set $X \subseteq 2^{\kappa}$ is a Borel ${ }^{*}$-set if there is a Borel ${ }^{*}$-code $(T, h)$ such that for all $\eta \in 2^{\kappa}, \eta \in X$ if and only if II has a winning strategy in the game $B^{*}(T, h, \eta)$.

Notice that a strategy in a game $B^{*}(T, h, \eta)$ can be seen as a function $\sigma: \kappa^{<\kappa} \rightarrow \kappa$, because every $\kappa^{+} \kappa$-tree can be seen as a downward closed subtree of $\kappa^{<\kappa}$.

Suppose $T$ is a closed $\kappa^{+}, \kappa$-tree and $h$ is a function with domain $T$ such that if $x \in T$ is a leaf, then $h(x)$ is a Borel set and otherwise $h(x) \in\{\cup, \cap\}$. Then the set

$$
\left\{\eta \mid \text { II has a winning strategy in the game } B^{*}(T, h, \eta)\right\}
$$

is a Borel*-set. This can be seen by the Borel*-code $\left(T^{\prime}, h^{\prime}\right)$, where $T^{\prime}$ is $T$ concatenated in every leaf, $b$, by the tree $T_{b}$ and $h^{\prime}$ is the union of the functions $h_{b}$, where $\left(T_{b}, h_{b}\right)$ is a Borel*-code for the set $h(b)$.

Theorem 3.8. Suppose that $\kappa=\kappa^{<\kappa}=\lambda^{+}, 2^{\lambda}>2^{\omega}$ and $\lambda^{<\lambda}=\lambda$. Then the following statements are consistent.

1. If $T_{1}$ is classifiable and $T_{2}$ is not, then there is an embedding of $(\mathcal{P}(\kappa), \subseteq)$ to $\left(B^{*}\left(T_{1}, T_{2}\right), \leqslant B\right)$, where $B^{*}\left(T_{1}, T_{2}\right)$ is the set of all Borel*-equivalence relations strictly between $\cong_{T_{1}}$ and $\cong_{T_{2}}$.

2. If $T_{1}$ is classifiable and $T_{2}$ is unstable or superstable, then

$$
\cong_{T_{1}}^{\kappa} \leqslant_{c} E_{\lambda-c l u b}^{2} \leqslant_{c} \cong_{T_{2}}^{\kappa} \wedge \cong_{T_{2}}^{\kappa} \mathbb{B}_{B} E_{\lambda-\text {-club }}^{2} \wedge E_{\lambda-c l u b}^{2} \nless_{B} \cong_{T_{1}}^{\kappa} .
$$

Proof. We will start the proof with two claims.

Claim 3.9. If $\diamond_{\kappa}(S)$ holds in $V$ and $\mathbb{Q}$ is $\kappa$-closed, then $\diamond_{\kappa}(S)$ holds in every $\mathbb{Q}$-generic extension.

Proof. Let us proceed by contradiction. Suppose $\left(S_{\alpha}\right)_{\alpha \in S}$ is a $\nabla_{\kappa}(S)$-sequence in $V$ but not in $V[G]$, for some generic $G$. Fix the names $\breve{S}, \dot{C}, \dot{X} \in V^{\mathbb{Q}}$ and $p \in G$, such that:

$$
p \Vdash\left(\dot{C} \subseteq \check{\kappa} \text { is a club } \wedge \dot{X} \subseteq \check{\kappa} \wedge \forall \alpha \in \dot{C}\left[\check{S}_{\alpha} \neq \dot{X} \cap \alpha\right]\right) .
$$

Working in $V$, we choose by recursion $p_{\alpha}, \beta_{\alpha}, \theta_{\alpha}$, and $\delta_{\alpha}$ such that:

1. $p_{\alpha} \in \mathbb{Q}, p_{0}=p$ and $p_{\alpha} \geqslant p_{\gamma}$ if $\alpha \leqslant \gamma$.

2. $\beta_{\alpha} \leqslant \beta_{\gamma}$ if $\alpha \leqslant \gamma$.

3. $\beta_{\alpha} \leqslant \theta_{\alpha}, \delta_{\alpha}<\beta_{\alpha+1}$.

4. If $\gamma$ is a limit ordinal, then $\beta_{\gamma}=\delta_{\gamma}=\cup_{\alpha<\gamma} \beta_{\alpha}$.

5. $p_{\alpha+1} \Vdash\left(\check{\delta}_{\alpha} \in \check{C} \wedge \dot{X} \cap \check{\beta}_{\alpha}=\check{S}_{\theta_{\alpha}}\right)$. 
We will show how to choose them such that 1-5 are satisfied. First, for the successor step assume that for some $\alpha<\kappa$ we have chosen $p_{\alpha+1}, \beta_{\alpha}, \theta_{\alpha}$ and $\delta_{\alpha}$. We choose any ordinal satisfying 3 as $\beta_{\alpha+1}$. Since $p_{\alpha+1} \Vdash\left(\dot{C} \subseteq \breve{\kappa}\right.$ is a club), there exists $q \in \mathbb{Q}$ stronger than $p_{\alpha+1}$ and $\delta<\kappa$ such that $q \Vdash\left(\check{\delta} \in \dot{C} \wedge \check{\beta}_{\alpha} \leqslant \check{\delta}\right)$. Now set $\delta_{\alpha+1}=\delta$. Since $\mathbb{Q}$ is $\kappa$-closed, there exists $Y \in \mathcal{P}\left(\beta_{\alpha+1}\right)^{V}$ and $r \in \mathbb{Q}$ stronger than $q$ such that $r \Vdash \dot{X} \cap \breve{\beta}_{\alpha+1}=\check{Y}$. By $\nabla_{\kappa}(S)$ in $V$, the set $\left\{\gamma<\kappa \mid Y=S_{\gamma}\right\}$ is stationary, so we can choose the least ordinal $\theta_{\alpha+1} \geqslant \beta_{\alpha+1}$ such that $r \Vdash \dot{X} \cap \breve{\beta}_{\alpha+1}=\check{S}_{\theta_{\alpha+1}}$. Clearly $r=p_{\alpha+2}$ satisfies 1 and 5 .

For the limit step, assume that for some limit ordinal $\alpha<\mathcal{k}$ we have chosen $p_{\gamma}, \beta_{\gamma}, \theta_{\gamma}$ and $\delta_{\gamma}$ for every $\gamma<\alpha$. Note that by 4 we know how to choose $\beta_{\alpha}$ and $\delta_{\alpha}$. Since $\mathbb{Q}$ is $\kappa$-closed, there exists $p_{\alpha}$ that satisfies 1 . We choose $\theta_{\alpha}$ as in the successor case with $q=p_{\alpha}$ and $p_{\alpha+1}$ as the condition $r$ used to choose $\theta_{\alpha}$.

Define $A, B$ and $C_{\delta}$ by $B=\cup_{\alpha<k} S_{\theta_{\alpha}}, A=\left\{\alpha \in S \mid B \cap \alpha=S_{\alpha}\right\}$ and $C_{\delta}=\left\{\delta_{\alpha} \mid \alpha\right.$ is a limit ordinal $\}$. Note that $C_{\delta}$ is a club. By $\nabla_{\kappa}(S)$ in $V, A$ is stationary and $A \cap C_{\delta} \neq \varnothing$. Let $\delta_{\alpha} \in A \cap C_{\delta}$. Then by 1,2 and 5, for every $\gamma>\alpha$ we have $p_{\gamma+1} \Vdash\left(\check{S}_{\theta_{\alpha}}=\check{S}_{\theta_{\gamma}} \cap \check{\beta}_{\alpha}\right)$. Therefore, $S_{\theta_{\alpha}}=B \cap \beta_{\alpha}$ and $\delta_{\alpha} \in A \cap C_{\delta}$ and so by 4 we have $S_{\theta_{\alpha}}=B \cap \delta_{\alpha}=S_{\delta_{\alpha}}$. But now by 5 we get $p_{\alpha+1} \Vdash\left(\check{\delta}_{\alpha} \in \check{C} \wedge \dot{X} \cap \check{\delta}_{\alpha}=\check{S}_{\delta_{\alpha}}\right)$ which is a contradiction.

Claim 3.10. For all stationary $X \subseteq \kappa$, the relation $E_{X}$ is a Borel*-set.

Proof. The idea is to code the club-game into the Borel*-game: in the club-game the players pick ordinals one after another and if the limit is in a predefined set $A$, then the second player wins. Define $T_{X}$ as the tree whose elements are all the increasing elements of $\kappa \leqslant \lambda$, ordered by end-extension. For every element of $T_{X}$ that is not a leaf, define

$$
H_{X}(x)= \begin{cases}\cup & \text { if } x \text { has an immediate predecessor } x^{-} \text {and } H_{X}\left(x^{-}\right)=\cap \\ \cap & \text { otherwise }\end{cases}
$$

and for every leaf $b$ define $H_{X}(b)$ by:

$$
(\eta, \xi) \in H_{X}(b) \Longleftrightarrow \text { for every } \alpha \in \lim (\operatorname{ran}(b)) \cap X(\eta(\alpha)=\xi(\alpha))
$$

where $\alpha \in \lim (\operatorname{ran}(b))$ if $\sup (\alpha \cap \operatorname{ran}(b))=\alpha$.

Let us assume there is a winning strategy $\sigma$ for Player II in the game $B^{*}\left(T_{X}, H_{X},(\eta, \xi)\right)$ and let us conclude that $(\eta, \xi) \in E_{X}$. Clearly by the definition of $H_{X}$ we know that $\eta$ and $\xi$ coincide in the set $B=\left\{\alpha<\mathcal{\kappa} \mid \sigma\left[\operatorname{dom}(\sigma) \cap \alpha^{<\lambda}\right] \subset \alpha^{<\lambda}\right\} \cap X$. Since $\lambda^{<\lambda}=\lambda$, we know that $B^{\prime}=\{\alpha<\kappa \mid$ $\left.\sigma\left[\operatorname{dom}(\sigma) \cap \alpha^{<\lambda}\right] \subset \alpha^{<\lambda}\right\}$ is closed and unbounded. Therefore, there exists a club that doesn't intersect $\left(\eta^{-1}[1] \triangle \xi^{-1}[1]\right) \cap X$.

For the other direction, assume that $\left(\eta^{-1}[1] \triangle \xi^{-1}[1]\right) \cap X$ is not stationary and denote by $C$ the club that does not intersect $\left(\eta^{-1}[1] \triangle \xi^{-1}[1]\right) \cap X$. The second player has a winning strategy for the game $B^{*}\left(T_{\lambda}, H_{X},(\eta, \xi)\right)$ : she makes sure that, if $b$ is the leaf in which the game ends and $A \subset \operatorname{ran}(b)$ is such that $\sup (\cup A) \in X$, then $\sup (\cup A) \in C$. This can be done by always choosing elements $f \in \kappa^{<\lambda}$ such that $\sup (\operatorname{ran}(f)) \in C$.

Let $\mathbb{P}$ be $\{f: X \rightarrow 2|X \subseteq \kappa| X \mid,<\kappa\}$ with the order $p \leqslant q$ if $q \subset p$. It is known that in any $\mathbb{P}$-generic extension, $V[G], \nabla_{\kappa}(S)$ holds for every $S \in V, S$ a stationary subset of $\kappa$.

1. In [FHK14, Thm 52] the following was proved under the assumption $\kappa=\lambda^{+}$and GCH:

For every $\mu<\kappa$ there is a $\kappa$-closed forcing notion $Q$ with the $\kappa^{+}$-c.c. which forces that there are stationary sets $K(A) \subsetneq S_{\mu}^{\kappa}$ for each $A \subsetneq \kappa$ such that $E_{K(A)} \nless_{B} E_{K(B)}$ if and only if $A \not \subset B$. 
In [FHK14, Thm 52] the proof starts by taking $\left(S_{i}\right)_{i<\kappa}, \kappa$ pairwise disjoint stationary subsets of $\lim \left(S_{\mu}^{\kappa}\right)=\left\{\alpha \in S_{\mu}^{\mathcal{K}} \mid \alpha\right.$ is a limit ordinal in $\left.S_{\mu}^{\mathcal{K}}\right\}$, and defining $K(A)=\cup_{\alpha \in A} S_{\alpha} . \mathbb{Q}$ is an iterated forcing that satisfies: For every name $\sigma$ of a function $f: 2^{\kappa} \rightarrow 2^{\kappa}$, exists $\beta<\kappa$ such that, $\mathbb{P}_{\beta} \Vdash$ " $\sigma$ is not a reduction".

With a small modification on the iteration, it is possible to construct $\mathbb{Q}$ a $\kappa$-closed forcing with the $\kappa^{+}$-c.c. that forces

(*) For $\mu \in\{\omega, \lambda\}$ and $A \subsetneq \kappa$, there are stationary sets $K(\mu, A) \subsetneq S_{\mu}^{\kappa}$ for which $E_{K(\mu, A)} \mathbb{B}_{B} E_{K(\mu, B)}$ if and only if $A \not \subset B$.

Without loss of generality we may assume that $\mathrm{GCH}$ holds in $V$. Let $G$ be a $\mathbb{P} * \mathrm{Q}$-generic. It is enough to prove that for every $A \subsetneq \kappa$ in $V[G]$ the following holds:

(a) If $T_{2}$ is unstable, or superstable with OTOP or with DOP, then $E_{K(\lambda, A)} \in B^{*}\left(T_{1}, T_{2}\right)$.

(b) If $T_{2}$ is stable unsuperstable, then $E_{K(\omega, A)} \in B^{*}\left(T_{1}, T_{2}\right)$.

In both cases the proof is the same; we will only consider (a).

Working in $V[G]$, let $T_{2}$ be as in (a). Since $\mathbb{Q}$ is $\kappa$-closed, we have $V[G] \models \diamond_{\kappa}(S)$ for every stationary $S \subset \kappa, S \in V$. Since $\mathbb{P}$ and $\mathbb{Q}$ are $\kappa$-closed and have the $\kappa^{+}$-c.c., we have $\kappa=\kappa^{<\kappa}=\lambda^{+}, 2^{\lambda}>2^{\omega}$ and $\lambda^{<\lambda}=\lambda$. By Lemma 2.3, Theorems 3.1 and 3.4, we have that $\cong_{T_{1}}^{\kappa} \leqslant_{c} E_{K(\lambda, A)} \leqslant_{c} \cong_{T_{2}}^{\kappa}$ holds for every $A \subsetneq \kappa$. The argument in the proof of Theorem 2.4 can be used to prove that $E_{K(\lambda, A)}{ }_{B} \cong_{T_{1}}^{\kappa}$ holds for every $A \subsetneq \kappa$.

To show that $\cong T_{T_{2}}^{\kappa} \mathbb{K}_{B} E_{K(\lambda, A)}$ holds for every $A \subsetneq \mathcal{K}$, assume towards a contradiction that there exists $B \subsetneq \kappa$ such that $\cong_{T_{2}}^{\kappa} \leqslant B E_{K(\lambda, B)}$. But then $E_{K(\lambda, A)} \leqslant_{B} E_{K(\lambda, B)}$ holds for every $A \subsetneq \kappa$ and by $(*), A \subsetneq B$ for every $A \subsetneq \kappa$. So $B=\kappa$ which is a contradiction.

2. In [HK12, Thm 3.1] it is proved (under the assumptions $2^{\kappa}=\kappa^{+}$and $\kappa=\kappa^{<\kappa}>\omega$ ) that there is a generic extension in which $\cong_{D L O}^{\kappa}$ is not a Borel ${ }^{*}$-set. The forcing is constructed using the following claim [HK12, Claim 3.1.5]:

For each $(t, h)$ there exists a $\kappa^{+}$-c.c. $\kappa$-closed forcing $\mathbb{R}(t, h)$ such that in any $\mathbb{R}(t, h)$-generic extension $\cong_{D L O}^{\kappa}$ is not a Borel $^{*}$-set.

The forcing in [HK12, Thm 3.1] works for every theory $T$ that is unstable, or $T$ non-classifiable and superstable (not only DLO, see [HK12] and [HT91]). Therefore, this claim can be generalized to:

For each $(t, h)$ there exists a $\kappa^{+}$-c.c. $\kappa$-closed forcing $\mathbb{R}(t, h)$ such that in any $\mathbb{R}(t, h)$-generic extension, $\cong_{T}^{\kappa}$ is not a Borel*-set, for all T unstable, or T non-classifiable and superstable.

By iterating this forcing (as in [HK12, Thm 3.1]), we construct a $\kappa$-closed forcing $\mathbb{Q}, \kappa^{+}$-c.c. that forces $\cong_{T}^{\kappa}$ is not a Borel $^{*}$-set, for all $T$ unstable, or $T$ non-classifiable and superstable.

Wwithout loss of generality we may assume that $2^{\kappa}=\kappa^{+}$holds in $V$. Let $G$ be a $\mathbb{P} * Q$-generic. Since $\mathbb{Q}$ is $\kappa$-closed, $V[G] \models \nabla_{\kappa}(S)$ for every stationary $S \subset \kappa, S \in V$. Since $\mathbb{P}$ and $\mathbb{Q}$ are $\kappa$-closed and have the $\kappa^{+}$-c.c., we have $\kappa=\kappa^{<\kappa}=\lambda^{+}, 2^{\lambda}>2^{\omega}$ and $\lambda^{<\lambda}=\lambda$. Working in $V[G]$, let $T_{2}$ be unstable, or non-classifiable and superstable. By Lemma 2.3, Theorems 3.3 and 3.4 we finally have that $\cong_{T_{1}}^{\kappa} \leqslant_{c} E_{\lambda \text {-club }}^{2} \leqslant_{c} \cong{ }_{T_{2}}^{\kappa}$ and $E_{\lambda \text {-club }}^{2} \gtrless_{B} \cong_{T_{1}}^{\kappa}$ holds.

Since $2^{\kappa} \times 2^{\kappa}$ is homeomorphic to $2^{\kappa}$, in order to finish the proof, it is enough to show that if $f: 2^{\kappa} \rightarrow 2^{\kappa}$ is Borel, then for all Borel*-sets $A$, the set $f^{-1}[A]$ is a Borel*. This is because if $f$ were the reduction $\cong_{T_{2}}^{\kappa} \leqslant B E_{\lambda \text {-club' }}^{2}$ we would have $(f \times f)^{-1}\left[E_{\lambda \text {-club }}^{2}\right]=\cong_{T_{2}}^{\kappa}$ and since $E_{\lambda \text {-club }}^{2}$ is Borel ${ }^{*}$, this would yield the latter Borel* as well. 
Claim 3.11. Assume $f: 2^{\kappa} \rightarrow 2^{\kappa}$ is a Borel function and $B \subset 2^{\kappa}$ is Borel*. Then $f^{-1}[B]$ is Borel ${ }^{*}$.

Proof. Let $\left(T_{B}, H_{B}\right)$ be a Borel ${ }^{*}$-code for $B$. Define the Borel*-code $\left(T_{A}, H_{A}\right)$ by letting $T_{B}=T_{A}$ and $H_{A}(b)=f^{-1}\left[H_{B}(b)\right]$ for every branch $b$ of $T_{B}$. Let $A$ be the Borel*-set coded by $\left(T_{A}, H_{A}\right)$. Clearly, II $\uparrow B^{*}\left(T_{B}, H_{B}, \eta\right)$ if and only if II $\uparrow B^{*}\left(T_{A}, H_{A}, f^{-1}(\eta)\right)$, so $f^{-1}[B]=A$.

We end this paper with an open question:

Question 3.12. Is it provable in ZFC that $\cong_{T}^{\kappa} \varlimsup_{B} \cong_{T^{\prime}}^{\mathcal{K}}$ (note the strict inequality) for all complete first-order theories $T$ and $T^{\prime}, T$ classifiable and $T^{\prime}$ not? How much can the cardinality assumptions on $\kappa$ be relaxed?

\section{References}

[FHK14] S.D. Friedman, T. Hyttinen, and V. Kulikov, Generalized descriptive set theory and classification theory, Memoirs of the Amer. Math. Soc. Vol. 230/1081 (American Mathematical Society, 2014).

[HK12] T. Hyttinen, and V. Kulikov, Borel* sets in the generalized Baire space - arXiv:1209.3933 [math.LO](2012)

[HM15] T. Hyttinen, and M. Moreno, On the reducibility of isomorphism relations - arXiv:1509.05291 [math.LO](2015).

[HT91] T. Hyttinen, and H. Tuuri, Constructing strongly equivalent nonisomorphic models for unstable theories, APAL 52 203-248, (1991).

[Kun11] K. Kunen, Set theory, Studies in Logic: Mathematical Logic and Foundations. Vol. 34, (College Publications, London, 2011).

[MV93] A. Mekler, and J. Väänänen, Trees and $\Pi_{1}^{1}$ subsets of $\omega_{1}^{\omega_{1}}$, J. Symb. Log. 58(3), 97-114, (1993).

[She90] S. Shelah, Classification theory, Stud. Logic Found. Math. Vol. 92, (North-Holland, Amsterdam, 1990).

[She10] S. Shelah, Diamonds, Proc. Amer. Math. Soc. 138, 2151-2161, (2010).

[Vau74] R. Vaught, Invariant sets in topology and logic, Fund. Math. 82, 269-294, (1974/75). 